\title{
Exploring the link between time to collision and representational momentum
}

\author{
Rob Gray, lan M Thornton $\uparrow$ \\ Cambridge Basic Research, Nissan Technical Center North America, Inc., Nissan CBR, 4 Cambridge \\ Center, Cambridge, MA 02142, USA; e-mails: rgray@cbr.com, ian.thornton@tuebingen.mpg.de \\ Received 7 February 2000, in revised form 10 December 2000
}

\begin{abstract}
Previous research has shown that when a moving object is occluded prior to collision with another stationary object, observers tend to consistently underestimate the physical/actual time to collision (TTC). We examined whether mislocalization of the initial point of disappearance plays any role in this underestimation. To assess the contribution of mislocalization, we coupled a standard TTC paradigm with a representational-momentum paradigm. Representational momentum refers to the tendency to mislocalize the remembered stopping point of a moving object as being too far forward along an actual or implied path of motion. Using identical displays we found large representational-momentum shifts and consistent underestimation of TTC. When the displays were modified to disambiguate the point of disappearance, representational momentum was absent or significantly reduced and underestimates of TTC were effectively eliminated. These results strongly suggest that the represented point of disappearance is an important factor in TTC estimation and that systematic forward displacement of this point may partially explain frequently observed underestimation.
\end{abstract}

\section{Introduction}

Almost every action we perform involves dealing with potential collisions. Everyday activities such as driving a car or walking across the road require collision avoidance. Interceptive actions such as grasping, hitting, and catching involve purposely engineering a collision. The ecological significance of dealing with collisions is reflected in the large amount of research on the perceptual information associated with impending collisions, namely information specifying the time to collision (TTC) of a moving object.

One common paradigm that has been used to investigate TTC involves presenting a moving object (simulated or real) for a brief period, occluding the object a variable time before collision and then asking the observer to respond at the point in time when he/she thinks collision would have occurred. Object motion can be towards the observer (ie motion in depth; Cavallo and Laurent 1988) or towards some fixed barrier (ie translation in the picture plane; Rosenbaum 1975). This paradigm is commonly called the predictive-motion (PM) paradigm (Tresilian 1995). Using the PM paradigm, researchers have found that the majority of observers underestimate the actual TTC. That is, the object is perceived to arrive sooner than it actually would have.

For motion-in-depth, large underestimates of TTC have been reported for simulated objects approaching a stationary observer (Schiff and Detwiler 1979), simulations of an observer moving on a collision course with a stationary object (McLeod and Ross 1983; Stewart et al 1993) and observers sitting in a car that is moving towards a stationary object (Cavallo and Laurent 1988). For motion in the picture plane, the accuracy of TTC estimates depends on the amount of time the moving object is occluded (Peterken et al 1991). For occlusion times less than $1 \mathrm{~s}$ observers can perform the PM task accurately with no consistent response bias (Rosenbaum 1975). For occlusion times greater than $1 \mathrm{~s}$, large underestimations of TTC have been reported (Peterken et al 1991). 
Several hypotheses have been put forward to explain the consistent underestimation of TTC. The most common of these proposes that humans have evolved an underestimation bias to ensure that we do not collide with objects. That is, if we must make errors in TTC estimation, it would seem better to err on the side of caution. However, this does not seem a likely explanation, given that observers do not consistently underestimate TTC in the more natural situation when binocular information about TTC is available (Gray and Regan 1998). In addition, making an avoidance response too early can be potentially dangerous because it can leave the observer vulnerable to other approaching objects and/or changes in the approaching object's trajectory or speed.

Bootsma and colleagues (Bootsma 1989; Savelsbergh et al 1991) have argued that large errors found in TTC estimation studies could result from decoupling perception and action. These authors propose that the perceptual processing involved in the performance of complex motor actions may only occur during the execution of these actions. Purely perceptual judgments of TTC may be much less accurate than judgments made while hitting or catching a ball, as the tight link between perception and action has been broken and is only maintained in the latter situations. While Bootsma (1989) was able to show that TTC errors were significantly smaller and less variable for direct actions (eg hitting a ball dropped from above) versus perceptual judgments (eg pressing a button when the ball reached a specific point), it is not clear that this hypothesis can explain why perceptual TTC errors are usually underestimations rather than overestimations.

Another approach has been to consider the nature of more general cognitive mechanisms that might underlie performance in the occlusion paradigm. The logic here is that, if systematic errors are usually associated with these mechanisms, then this might help explain the observed underestimation of TTC. DeLucia and Liddell (1998), among others, have suggested that performance in TTC tasks may rely on some form of cognitive motion extrapolation (eg Cooper 1989; Finke and Shyi 1988; Jagacinski et al 1983; Rosenbaum 1975) and/or some form of internal clocking or timing mechanism (eg Lyon and Waag 1995; Tresilian 1995). As there is evidence in the literature for both timing errors (eg Zackay and Block 1997, for a review) and extrapolation errors (eg Cooper 1989; Finke and Shyi 1988; Hayes and Thornton 1999) which would be consistent with underestimated TTC, this approach may be effective for understanding TTC underestimation. As yet, however, there is little direct evidence linking TTC to these more general cognitive mechanisms.

In the current work, we take a similar approach, but, instead of focusing on the cognitive mechanisms themselves, we explore the nature of the mental representations that might underlie computations of TTC. More specifically, we ask whether systematic mislocalization of where the moving object actually disappears may contribute to the typically observed underestimation. Freyd (eg 1987, 1992) has argued that objects undergoing real or implied motion may give rise to what she has called dynamic mental representations. That is, representations that are specifically tuned to capture spatiotemporally coherent change over time. One important characteristic of this type of representation is the phenomenon known as representational momentum (Freyd and Finke 1984).

Representational momentum refers to the consistent finding that memory for the final location of an object undergoing real or implied motion is systematically distorted or shifted 'forward' along the path of motion. Taking an analogy from physics, the suggestion is that the dynamic mental representation of a motion event, in directly modeling change over time, comes to possess momentum. When such representations are accessed, the represented dynamics cannot be immediately stopped, much in the same way that a moving physical object will not come to rest as soon as a resisting force is applied, so memory for the final position is distorted. While early work attempted 
to explore direct links between physical and representational momentum (eg Finke et al 1986), more recent efforts have focused instead on representational momentum being a general consequence of spatiotemporal coherence, predicting that anticipatory computations might lead to representational momentum along any dimension of continuous change (Freyd 1992; see also Brehaut and Tipper 1996, and Hubbard 1995a for further discussion of this issue).

The forward memory shifts associated with representational momentum have been observed across a variety of stimulus types and display configurations including horizontal (Thornton et al 1996), vertical (Hubbard and Bharucha 1988), curvilinear (Freyd and Jones 1994), circular (Hubbard 1996), and oblique (Hubbard 1990) translation in the picture plane, approaching and receding translation in depth, both in 2-D environments (Hubbard 1995b; Kelly and Freyd 1987) and 3-D environments (Hayes et al 1996), as well as rotation, both in the picture plane (eg Freyd and Finke 1984, 1985) and in depth (Munger et al 1999). This phenomenon does not appear to be susceptible to training or feedback (Freyd and Finke 1985) but can be influenced by a number of factors such as velocity (Freyd and Finke 1985), acceleration (Finke et al 1986), retention interval (Freyd and Johnson 1987), attention (Hayes 1997), expectation (Hubbard and Bharucha 1988; Reed and Vinson 1996; Verfaillie and d'Ydewalle 1991), and surrounding context (Brehaut 1989; Hubbard 1993; Hubbard and Ruppel 1999; Thornton et al 1996).

There are many different perceptual strategies that have been put forth to explain how observers might perform the TTC judgment in the PM paradigm (see Gray and Regan 1999a; Tresilian 1995 for discussion). We now consider how the point of disappearance might influence three of these strategies: (i) the tau $(\tau)$ strategy, (ii) the distance/velocity strategy, and (iii) motion extrapolation.

The most popular account of TTC estimation is that judgments of TTC are based on the rate of change of size of an object directly approaching the eye (ie $\tau$, Lee 1976) and/or the rate of constriction of the visual gap between the object and the point of collision for objects not on a direct approach (Bootsma and Oujedans 1993). For example, in figure 1, the TTC is approximately equal to the instantaneous angular separation $(\theta)$ between the moving target and the barrier, divided by the rate at which this separation is changing $(\mathrm{d} \theta / \mathrm{d} t)$. Tresilian (1995) proposed that, in the PM paradigm, observers may initially estimate TTC on the basis of this ratio and then use an internal clocking process to 'count down' time after the target disappears. How would a forward

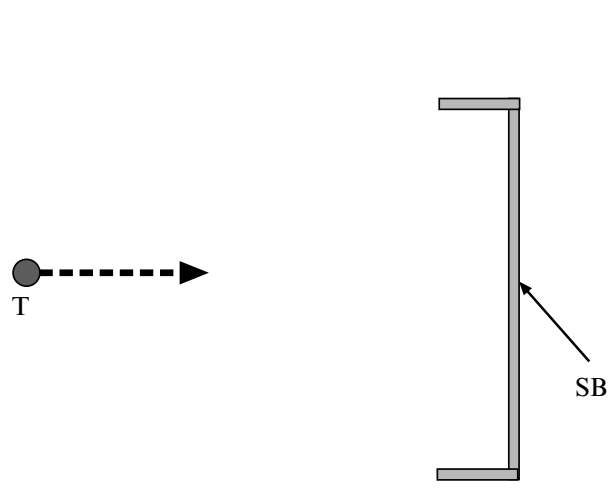

(a)

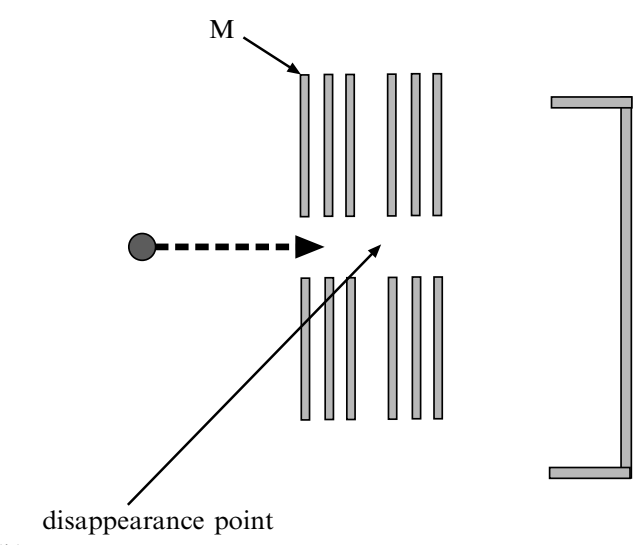

(b)

Figure 1. Visual display used in experiment 1. A small circular target (T) traveled towards a stationary barrier (SB) and disappeared a variable distance from the barrier. (a) Unmarked condition. (b) Marked condition in which stationary markers (M) were centered around the point of target disappearance. The markers appeared $150 \mathrm{~ms}$ prior to the instant of target disappearance. See text for details. 
shift in the position of the disappearance point affect the use of this strategy? First, it is reasonable to assume that observers will continually update their estimate of TTC on the basis of the ratio $\theta /(\mathrm{d} \theta / \mathrm{d} t)$ until the target disappears. The forward shift of the disappearance point would decrease the final value of $\theta$ without affecting the value of $\mathrm{d} \theta / \mathrm{d} t$; therefore, estimated TTC would be less than real TTC (ie an underestimation).

An alternative account of TTC estimation is that observers base their estimates of TTC on the ratio of perceived distance (ie between the object and the barrier) to perceived object speed (eg Cavallo and Laurent 1988). In the PM paradigm, performance of the task would involve estimating TTC on the basis of the distance/velocity ratio, then counting down time as described above. In this case the memory distortion for the disappearance point would cause distance to be underestimated presumably without affecting speed, resulting in a smaller value of TTC.

Thirdly, what would we expect if observers mentally extrapolated the motion of the target after it disappeared instead of generating an estimate of TTC? In this case, the forward shift of the target position would presumably jump the extrapolation ahead along the path of motion causing the observer to indicate a collision slightly earlier than the actual time of contact.

In the current work, we explore whether mislocalization of the point at which a moving object disappears contributes to underestimation in a TTC task. Clearly, if the internal representation of the point of disappearance is distorted forward in the direction of motion, as predicted by representational momentum, then, regardless of the mechanism(s) that use this representation (ie timing, extrapolation, etc), arrival time will be underestimated. In two experiments, we use identical displays to measure both TTC and representational momentum. In both experiments, when representational momentum is present, TTC is significantly underestimated for the majority of our observers. However, when the displays are modified so that the point of disappearance is disambiguated, representational momentum is significantly reduced and TTC underestimation disappears.

\section{Experiment 1}

\subsection{Purpose}

We compared measurements of representational momentum and estimates of TTC for identical visual displays in which (a) the moving target traveled across a blank field towards a stationary barrier or (b) stationary markers were flashed briefly around the point of disappearance of the moving target. In previous work, Thornton et al (1996) found that the addition of such markers during a previously blank retention interval significantly reduced observed forward shifts. As it is also possible that this manipulation may have affected the perceived speed of the moving target, we examined this issue more closely in experiment 2 below.

\subsection{Methods}

2.2.1 Apparatus. Stimuli were presented on an Indigo ${ }^{(i 0}$ workstation (Silicon Graphics Inc.) that was viewed binocularly from a distance of $57 \mathrm{~cm}$. The display was viewed in a dimly lit room so that the edges of the computer monitor were visible. The arrangement of the visual display is depicted in figure 1a. The moving target was a small (0.6 deg diameter) red circle. The target had a mean luminance of $20 \mathrm{~cd} \mathrm{~m}^{-2}$. The target moved from the observer's left to right towards a vertically oriented green barrier. The barrier subtended $18 \mathrm{deg}$ vertical $\times 0.25 \mathrm{deg}$ horizontal and had small ( $2.5 \mathrm{deg}$ horizontal $\times 0.25 \mathrm{deg}$ vertical) horizontally oriented arms attached to its ends.

2.2.2 Procedure. We first discuss the unmarked condition. Each trial consisted of one presentation of the moving target. The initial separation between the target and barrier, angular separation at the point of disappearance, and speed on each trial were chosen 
randomly from the following values: initial separation $(15.5,13.5$, and $11.5 \mathrm{deg})$, separation at disappearance $(9.4,7.7$, and $3.9 \mathrm{deg})$, and speed $\left(3.8,3.1\right.$, and $\left.2.5 \mathrm{deg} \mathrm{s}^{-1}\right)$.

For the representational-momentum task, the barrier alone was presented for $300 \mathrm{~ms}$ after the target disappeared. After this period, a red circular probe ( $0.6 \mathrm{deg}$ diameter $)$ and the barrier were presented for $150 \mathrm{~ms}$. The rationale for the barrier remaining visible for the entire duration of the trial was to keep the TTC and representational-momentum tasks as similar as possible (see below). The offset between the location of the probe position and the disappearance point of the moving target was chosen randomly from one of seven values $(0.9,0.6,0.3,0,-0.3,-0.6$, and $-0.9 \mathrm{deg}$ where positive values indicate offsets to the observer's right and negative values indicate offsets to the observer's left). The observer's task was to indicate whether the probe appeared in the same location as the disappearance point of the moving target or in a different location (either left or right) by pressing one of two response keys. Each probe location was repeated 10 times giving a total of 70 trials per run. The intertrial interval was $500 \mathrm{~ms}$.

For the TTC task, the barrier remained on the screen after the moving target disappeared (as was the case in the majority of previous TTC experiments). The observer's task was to press the space bar when she/he felt that the target would have collided with the leftmost edge of the barrier assuming that the target continued to travel at the same speed after it disappeared.(1) The actual TTC for any given trial depended on the particular combination of speed and initial position (chosen randomly from trial to trial). TTC values (as measured from the point of disappearance) ranged from 1.55 to $3.7 \mathrm{~s}$. To allow for comparison, each TTC run also consisted of 70 trials.

In the marked condition, we presented stationary markers centered around the disappearance point of the moving target. The markers consisted of eight small (6.7 deg vertical $\times 0.25 \mathrm{deg}$ horizontal) bars as illustrated in figure $1 \mathrm{~b}$. The markers appeared on the screen $150 \mathrm{~ms}$ prior to the instant of disappearance of the moving target and remained on the screen until the target disappeared. Otherwise, all aspects of the two experimental tasks were as described above.

2.2.3 Observers. Twenty observers participated in experiment 1 . All observers were naïve to the aims of the experiment and were paid an hourly rate.

\subsection{Results}

2.3.1 Representational momentum. The results of the representational-momentum task are shown in figure 2. This figure shows the distribution of same responses as a function of probe location for both the unmarked and the marked conditions. In the unmarked condition, participants responded "same" on approximately $39 \%$ of trials. As there were only $14 \%$ of trials where the probe was physically in the same location as the true stopping point (probe 0), the majority of these responses are errors. What is of interest is the way in which these errors are distributed across the probe locations. It is clear from figure 2 that the tendency to endorse forward probe locations as being the same was considerably larger than the tendency to endorse backwards probe locations. Such a forward-biased distribution is the classic hallmark of representational momentum.

(1) Gray and Regan (1998) recently developed a staircase tracking method that, in general, produces more accurate and reliable estimates of TTC than tasks which require the observer to press a response key at the perceived time of collision. One of the main advantages of the staircase procedure is that it removes the effect of motor delay on the TTC estimate. We did not use this staircase procedure in the present study because we wanted to replicate the TTC paradigm used in the majority of previous research. We also expected the effect of motor delay would be similar for the unmarked and marked conditions in the present study. Finally, it should also be noted that the majority of observers still produce underestimates of TTC (albeit much smaller) when the staircase procedure is used. 


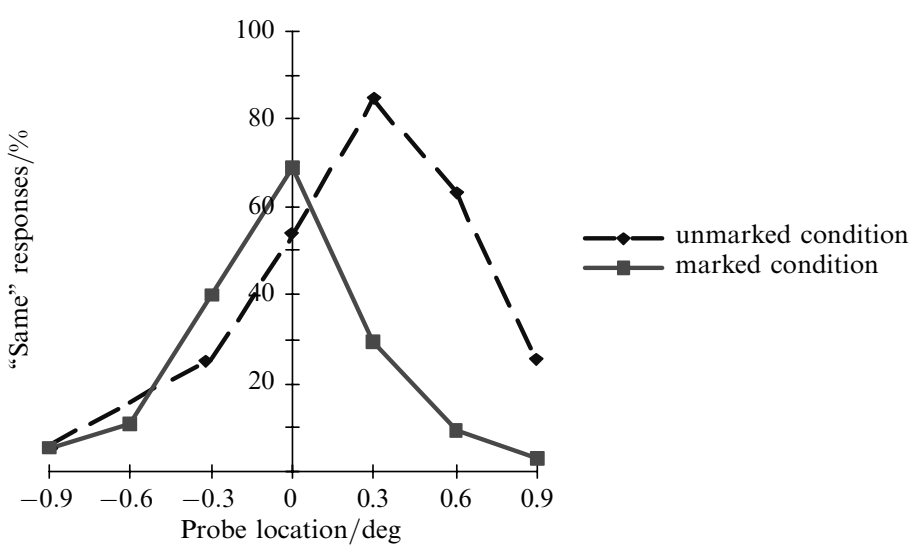

Figure 2. Results of the representational-momentum task for experiment 1. Data show the proportion of "same" responses as a function of probe position averaged across all observers. The 0 probe position is the true stopping point. It is clear that in the unmarked case the subjective stopping point is shifted forward. This bias is not apparent in the unmarked condition.

To quantify this bias, a central tendency was estimated for each participant by a weighted mean calculation (Faust 1990; Hayes 1997).(2) This analysis revealed that the estimated stopping point was, on average, some 0.26 deg further forward in the direction of motion than the true stopping point. This bias was consistently greater than zero $\left(M=0.26, t_{19}=7.35, p<0.05\right)$.

When the point of disappearance was marked, participants were generally less likely to respond "same". This is reflected in the overall shrinking of the same distribution for the marked condition, with "same" responses occurring only on $24 \%$ of trials. More importantly, participants were much more likely to restrict these "same" responses to the probes occurring at the true stopping point (probe 0). Not only is the central tendency of the marked distribution not different from zero $\left(M=-0.047, t_{19}=1.29\right.$, $p>1$ ), but the spread of responses around this weighted mean was reduced relative to the unmarked condition. The variance of same responses was computed by using an absolute average deviation measure (Hayes 1997). ${ }^{(3)}$ As the measure of central tendency employed in these studies is a weighted mean, we felt it more appropriate to use a measure of variance that was based on a similar weighting scheme. This is what the average absolute deviation measure introduced by Hayes (1997) is designed to do. On a subject-by-subject basis it calculates how tightly the same responses cluster around the central tendency. This revealed that the distribution for the unmarked condition $(M=0.31)$ was considerably more variable than for the marked condition $\left(M=0.20, t_{19}=5.48, p<0.05\right)$.

Taken together, the results of the representational-momentum task suggest that participants are able to use the disambiguating marking frame to localize the true

(2) The weighted mean for each observer is calculated by multiplying the proportion of "same" responses at a given probe position by that probe's distance from the true/same probe (ie 0 ). These products are then added and divided by the total number of "same" responses to yield a weighted mean.

(3) Absolute average deviation from the weighted mean was used as a measure of variance (Hayes 1997; Prinzmetal and Wilson 1997). This was computed for each observer simply by taking the absolute distance of each probe position from that observer's weighted mean, multiplying through by the number of "same" responses at that probe distance, summing these products and then dividing by the total number of "same" responses. The absolute average deviation thus provides a measure of variance based on our specific measure of central tendency. In general, absolute average deviation tends to be more robust to violations of parametric assumptions than the standard deviation (Keppel 1991). 
stopping point much more accurately. While the overall reduction in "same" responses may simply reflect a shift in criterion for saying "same" (perhaps reflecting uncertainty due to noise created by the briefly presented marking frame), the lack of bias and reduction in variability for the marked condition argue quite strongly that representational momentum is being eliminated in this condition. ${ }^{(4)}$

2.3.2 Time to collision. Consistent with previous findings (eg Schiff and Detwiler 1979), the majority $(15 / 20)$ of our observers underestimated TTC in the unmarked condition. Estimation errors for these fifteen observers ranged from $0.8 \%$ to $23 \%(M=6 \%)$ of the true TTC. As shown in figure 3a, when the point of disappearance is disambiguated, this underestimation almost completely disappears $(M=0.3 \%)$. The effect of this display manipulation was highly consistent, with all fifteen of these observers producing less extended estimates of TTC in the marked condition.

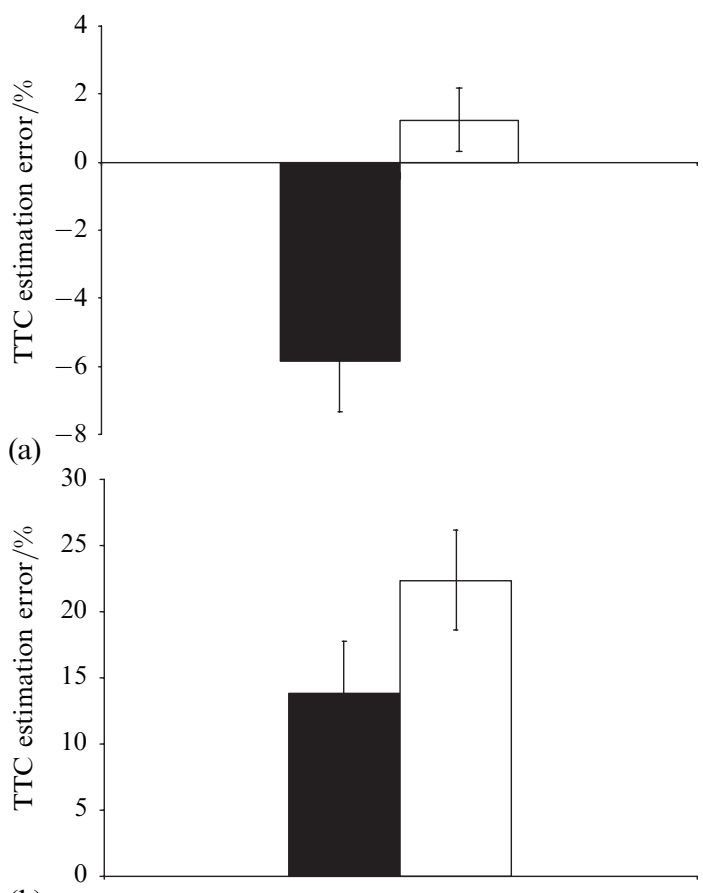

- unmarked condition

$\checkmark$ marked condition
Figure 3. Results of the time-to-collision (TTC) estimation task for experiment 1. Data are percentage errors in TTC estimation. Negative percentage errors represent underestimations while positive errors represent overestimations. (a) Data for fifteen observers that underestimated TTC. (b) Data for five observers that overestimated TTC. Error bars are standard errors.

(b)

The remaining five observers always overestimated TTC as shown in figure $3 \mathrm{~b}$. Overestimation for these observers in the unmarked condition was quite large, ranging from $6 \%$ to $24 \%(M=14 \%)$ and all five produced even larger overestimates when the point of disappearance was marked $(M=22 \%)$. Thus, all twenty observers produced estimates of TTC that were longer in the marked condition than in the unmarked condition. The mean shift in the percentage of TTC error, collapsed across all twenty observers was approximately $7.2 \%$, a figure that was significantly different from zero $\left(t_{19}=5.3, p<0.05\right)$.

(4) In previous studies of representational momentum velocity effects have been reported where increased actual or implied speed is accompanied by increased forward bias (Freyd and Finke 1985; Hubbard 1990, 1996; Hubbard and Bharucha 1988). We observed similar trends in the unmarked conditions of the current studies. Such trends were not present in the marked conditions. However, as velocity varied randomly within a given block of trials, detailed analyses of these trends were not possible. As velocity effects per se are not the main focus of this paper, data are reported collapsed across this factor. 
In the TTC task observers were instructed to press the bar at the perceived instant of the collision between the target and the barrier. However, it is also possible that they may have used an alternate strategy such as pressing the space bar a constant time interval after the point of disappearance. In any TTC task it is important to determine that observers based their responses on the task-relevant variable (ie the time to collision, TTC) rather than any of the task-irrelevant variables such as the speed or initial position (Gray and Regan 1998). To evaluate the relative weighting observers placed on the perceptual variables in the present experiment, we submitted the response data to a stepwise regression analysis with speed, initial position, final position, and TTC as independent variables. For all twenty observers TTC explained the largest amount of response variance. In the unmarked condition $R^{2}$ ranged from 0.62 to 0.84 , while in the marked condition $R^{2}$ ranged from 0.61 to 0.89 .

\subsection{Discussion}

When an identical baseline display was used, memory for the initial point of disappearance was consistently shifted forward in the direction of motion, and TTC estimates for the majority of observers were consistently underestimates. Of more interest, when the display was modified so that the point of disappearance was marked by a briefly presented reference frame, representational-momentum shifts completely vanished and TTC estimates were consistently lengthened.

The observed pattern of data appears consistent with our main hypothesis that both representational-momentum and TTC estimates rely on an internal representation of the point of disappearance. If such a representation were shifted forward, this would produce a forward bias in the distribution of same responses and underestimates of TTC. If observers are presented with additional information that disambiguates the point of disappearance, then we should predict a reduction in the forward bias and a reduction in the tendency to underestimate TTC. This is exactly the pattern observed.

The results of experiment 1 cannot rule out the possibility that our display manipulation flashing a disambiguating reference frame does more than simply mark the remembered point of disappearance. Moreover, any side effects of the display manipulation may well have different impacts on the two tasks we are examining. One possible side effect is that the flashed reference frame causes a shift of focal attention. Perhaps a more general side effect is simply to assume that the appearance of the flashed marker causes some temporary interruption in ongoing processing. Both of these alternative explanations arise because of the particular way we chose to disambiguate the point of disappearance. In experiment 2, we replicate the current design but replace the transient-marking frame with a constant, static reference frame.

\section{Experiment 2}

\subsection{Purpose}

In experiment 2, we used exactly the same design as for experiment 1 , but replaced the briefly presented marking frame with a constantly presented reference frame. As described in more detail below, the unmarked condition for both representationalmomentum and TTC blocks involved a single object moving towards a barrier through a smooth tube (figure 4). Our prediction was for strong representational-momentum shifts and consistent underestimation of TTC in this condition. The new marked condition was identical except that the tube was now demarcated with a series of teeth appearing at regular intervals. The purpose of the teeth was to provide a reference frame that would help to disambiguate the point of disappearance. Our prediction was that the marked condition would remove representational-momentum shifts and reduce the tendency to underestimate TTC. 


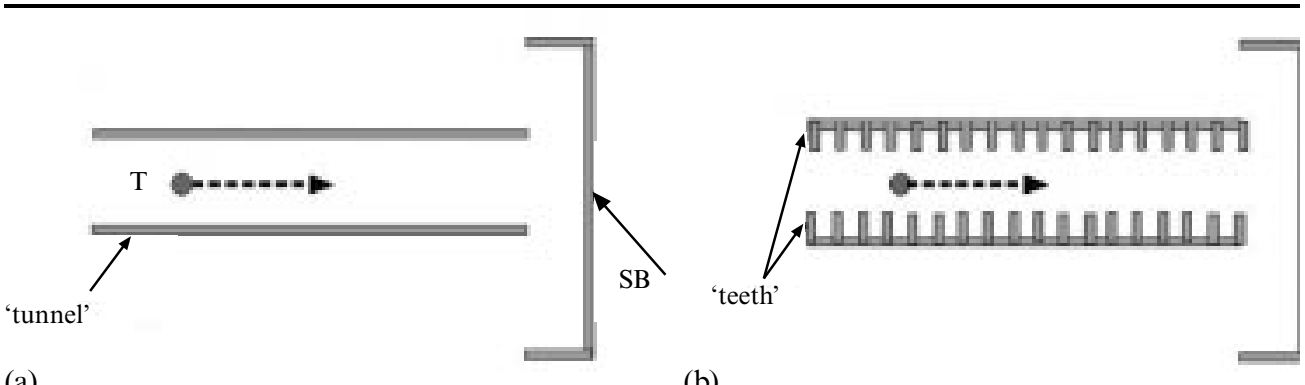

(a)

(b)

Figure 4. Visual display used in experiment 2. A small circular target (T) traveled towards a stationary barrier (SB) and disappeared a variable distance from the barrier. (a) Unmarked condition in which the moving target traveled through a 'tunnel' made up of two horizontally oriented bars. (b) Marked condition in which small 'teeth' were added to the 'tunnel'. See text for details.

As the marked condition no longer involved a disrupting transient event, we also hoped to rule out coincidental side effects such as attention shifting or motion disruption that might also influence TTC estimates. With these alternative influences ruled out, any change in TTC estimates between the unmarked and marked conditions should only be a consequence of the remembered point of disappearance. We thus predicted a clearer link between measured representational shifts and changes in TTC for each individual observer.

\subsection{Methods}

3.2.1 Procedure. The procedure was as described for experiment 1 except for the following. In the unmarked condition, the target traveled down the center of a tunnel consisting of two horizontally oriented bars as illustrated in figure 4a. The bars were green and subtended $21.2 \mathrm{deg}$ horizontal $\times 0.25 \mathrm{deg}$ vertical. In the marked condition, the display was identical except that small $(0.8 \mathrm{deg}$ vertical $\times 0.3 \mathrm{deg}$ horizontal $)$ vertically oriented teeth were attached to the two bars as shown in figure $4 \mathrm{~b}$. The 'teeth' were separated by $0.5 \mathrm{deg}$. We expected that the teeth would help observers localize the point of disappearance of the moving target within the tunnel.

For the representational-momentum task, the tunnel (and teeth in the marked condition) remained on the screen while the target was visible and for $300 \mathrm{~ms}$ after the target disappeared. The tunnel (and teeth) were not visible during the presentation of the probe. For the TTC task, the tunnel (and teeth) remained on the screen until the observer pressed the space bar to indicate perceived collision.

3.2.2 Observers. Twelve observers participated in experiment 2. All observers were naive to the aims of the experiment, were paid an hourly rate, and did not participate in experiment 1.

\subsection{Results}

3.3.1 Representational momentum. Figure 5 shows the results of the representationalmomentum task for both the unmarked and the marked conditions. As in experiment 1 , responses in the unmarked condition are clearly biased towards endorsing forward probe positions $(M=0.14)$, although the magnitude of this bias is somewhat reduced. In the marked condition, however, there is again very little bias $(M=0.02)$, and a direct comparison revealed that, as intended, marking the point of disappearance significantly reduced the size of the forward shift $\left(t_{11}=3.54, p<0.01\right)$. The forward bias in the unmarked condition was again consistently greater than zero $\left(t_{11}=2.7, p<0.05\right)$, whereas the shift in the marked condition was not consistently different from zero $\left(t_{11}=0.47, p>0.05\right)$. 


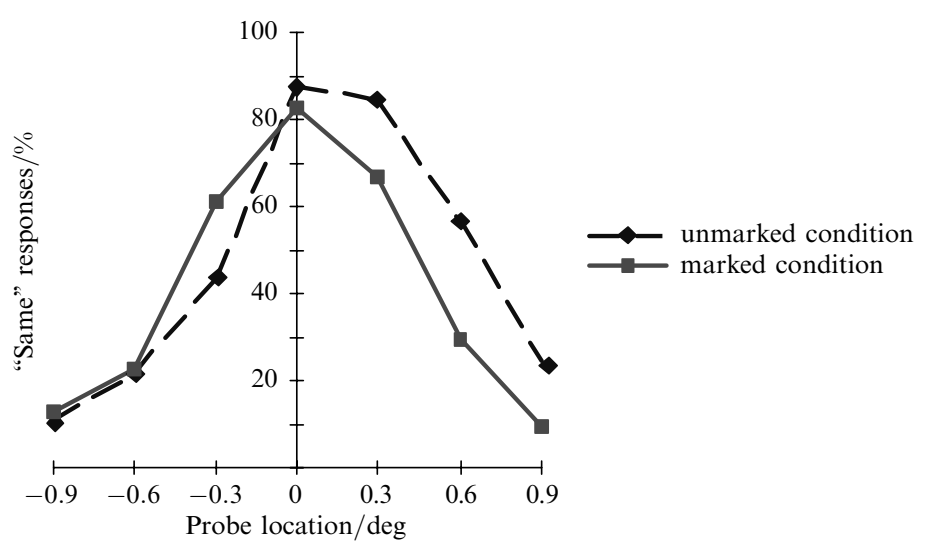

Figure 5. Results of the representational-momentum task for experiment 2. Data show the proportion of "same" responses as a function of probe position averaged across all observers. The 0 probe position is the true stopping point. It is clear that in the unmarked case the subjective topping point is shifted forward. This bias is not apparent in the unmarked condition.

As in experiment 1 , marking the point of disappearance also reduced the variance in these distributions, with the unmarked distribution $(M=0.31)$ being generally less variable than the marked distribution $(M=0.29)$. Here, however, this trend was only marginally significant $\left(t_{11}=1.49, p=0.08\right)$. In general, participants in experiment 2 were a little more liberal with their "same" responses, indicating no difference on $44 \%$ and $40 \%$ of trials for the unmarked and marked conditions, respectively. While the drop in the proportion of "same" responses was again significantly different $\left(t_{11}=2.39\right.$, $p<0.05$ ), it is far less dramatic than in experiment 1, suggesting that the flashed marker was the main cause of the large criterion shift in that experiment.

3.3.2 Time-to-collision estimation. Figures $6 \mathrm{a}$ and $6 \mathrm{~b}$ show the TTC estimation errors for the unmarked and marked conditions used in experiment 2. As was the case in

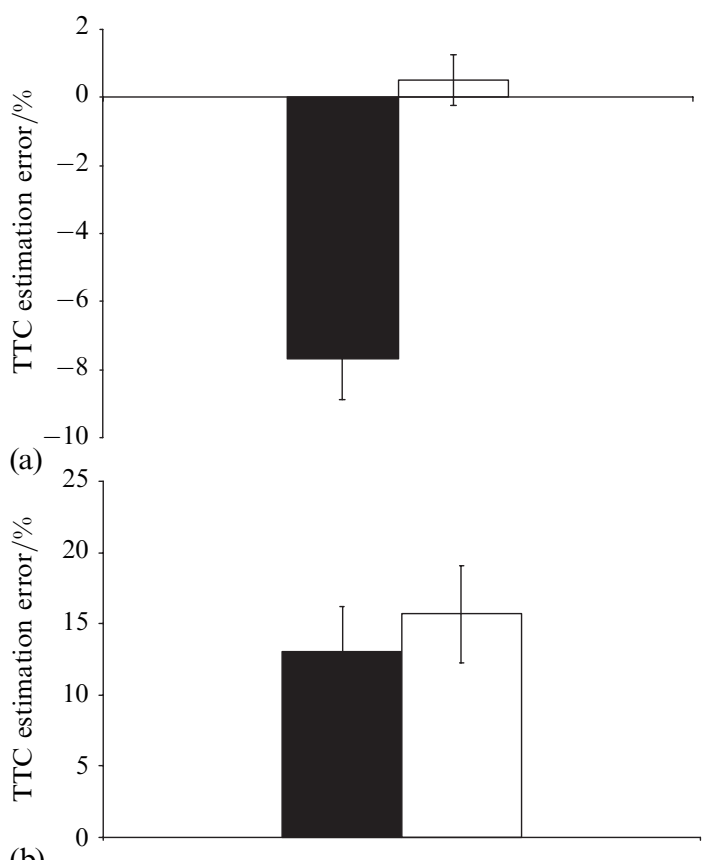

- unmarked condition

a marked condition

Figure 6. Results of the time-to-collision (TTC) estimation task for experiment 2. Data are percentage errors in TTC estimation. Negative percentage errors represent underestimations while positive errors represent overestimations. (a) Data for nine observers that underestimated TTC. (b) Data for three observers that overestimated TTC. Error bars are standard errors. 
experiment 1 , the majority of our observers (9/12) underestimated TTC in the unmarked condition (figure 6a). For $8 / 9$ of these underestimators the TTC judgment was more accurate in the marked condition than in the unmarked condition. Furthermore, for all twelve observers the estimated TTC was longer in the marked condition than in the unmarked condition. The mean shift in TTC estimation error $(M=5.3 \%)$ was again significantly different from zero $\left(t_{11}=5.9, p<0.001\right)$.

The results of the stepwise regression analysis performed on the experiment 2 data were also very similar to those found in experiment 1 in that the TTC explained a large amount of response variance $\left(R^{2}\right.$ ranged from 0.7 to 0.9 in the unmarked condition and from 0.72 to 0.87 in the marked condition). As in experiment 1 , the observed shift in TTC estimates $(M=5.3 \%)$ was of the same order of magnitude as the average shift predicted from the representational-momentum results $(M=3.1 \%)$.

\subsection{Discussion}

3.4.1 Linking representational momentum and time to collision. The two sets of analyses presented above clearly show that representational momentum and TTC can be strongly influenced by the same display manipulations. That is, forward displacements in the representational task and understimation in the TTC task were both decreased when the point of disappearance was disambiguated. These findings are certainly consistent with our main hypothesis that both effects typically involve biased representations of the true disappearance point.

Is there any further evidence in the data to support such a link? A further indication of a relationship between representational momentum and TTC would be to see clear patterns in the data of individual observers. For example, do participants with large representational-momentum shifts also have greater lengthening of TTC when the disappearance point is marked? To investigate this possibility, correlation coefficients between (a) unmarked weighted means and unmarked TTC estimates, and (b) unmarked weighted means and the observed change in TTC estimates (ie the difference between unmarked and marked TTC estimates) were calculated.

The former analysis reflects the impact that representational momentum might have on initial TTC estimates. There was a weak positive correlation $(r=0.39)$, such that larger representational-momentum shifts tended to be accompanied by larger underestimates. As noted in the introduction, we are not proposing that shifts in the remembered point of disappearance are the sole cause of TTC underestimates.

We can also ask whether the change in TTC can be predicted from the initial representational-momentum shifts? If, as discussed in section 1, TTC estimates rely on localization of the disappearance point, then we might expect the change in TTC estimation produced by reducing the mislocalization of the disappearance point to be related to the intial size of the mislocalization. Figure 7 shows the relationship between initial representational-momentum shifts and the observed change in TTC estimates. There was a strong positive correlation $(r=0.64)$ between the initial size of the weighted mean (ie the magnitude of the representational momentum in the unmarked condition) and the observed lengthening of TTC estimates (ie reduction in underestimation). ${ }^{(5)}$

(5) Similar, albeit weaker, links between TTC estimates and representational momentum were found for the data from experiment 1 . There was a weak positive correlation $(r=0.16)$ between the unmarked weighted means and unmarked TTC estimates. For the relationship between unmarked weighted means and the observed change in TTC estimates participants appear to cluster into two main groups. One group $(N=11)$, showed fairly large reduction in TTC $(M=12 \%)$ and another $(N=9)$, fairly modest reduction $(M=2 \%)$. Computing correlation coefficients within the two main groups reveals reasonably strong relationships between the size of the weighted mean and the reduction in TTC, with values of $r=0.74$ and 0.38 for the smaller and larger reduction groups, respectively. 


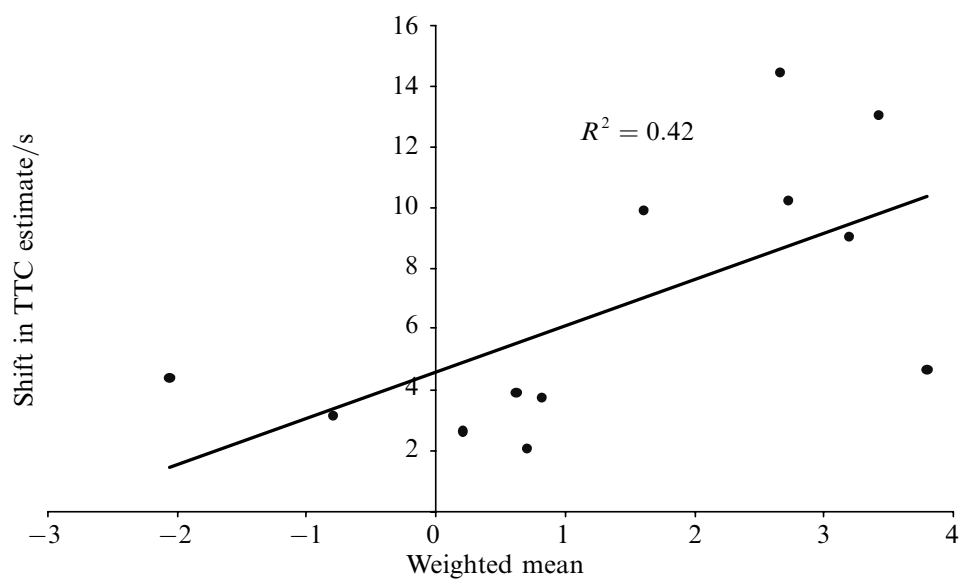

Figure 7. Relationship between initial representational and observed reduction in TTC.

3.4.2 Perceived speed. One concern in interpreting the results of experiment 2, was whether the lengthening of TTC in the marked condition was caused by changes in perceived speed. Previous research has shown that perceived speed is greatly influenced by the presence/absence of stationary reference marks around a moving target (Brown 1931; Gogel and McNulty 1983). Could changes in perceived speed caused by the marking 'teeth' explain the lengthening of TTC? Evidence against this explanation is as follows. First, our display manipulation had only a small effect on perceived speed. In a control experiment, we compared judgments of speed in the unmarked and marked conditions using a 2AFC design. Each presentation consisted of one interval with the marked display and one interval with the unmarked display. The order of the two displays was randomized. The target in the unmarked display interval always had a speed of $3.1 \mathrm{deg} \mathrm{s}^{-1}$ while the target in the marked display interval was chosen randomly from one of 8 values (equally spaced between $2.3 \mathrm{deg} \mathrm{s}^{-1}$ and $3.9 \mathrm{deg} \mathrm{s}^{-1}$ ). The observer's task was to indicate whether the target in the second interval moved faster or slower than the target in the first interval. The mean point of subjective equality (ie $50 \%$ faster responses) was $2.9 \mathrm{deg} \mathrm{s}^{-1}$. That is, on average the target in the marked display interval had to travel roughly $2.9 \mathrm{deg} \mathrm{s}^{-1}$ to be perceived to have the same speed as the target in the unmarked display interval moving at $3.1 \mathrm{deg} \mathrm{s}^{-1}$. This $3.8 \%$ increase in perceived speed for the marked condition is (a) in the wrong direction to explain the observed changes in TTC estimates and (b) is roughly half as large as the mean observed TTC shift. Second, perceived speed is higher the greater the number of neighboring elements (Gogel and McNulty 1983) so that we would expect to find larger underestimates of TTC in the marked condition if speed were a factor (ie the opposite pattern of the results shown in figure 6).

The results of experiment 2 generally replicate those of experiment 1 . In the baseline conditions the majority of observers underestimated TTC and showed fairly strong representational-momentum shifts. When a series of reference points was added in the marked condition, representational momentum disappeared and estimates of TTC were consistently lengthened. We had suggested that the flashed marking frame used in experiment 1 had side effects in addition to disambiguating the point of disappearance. Specifically, we were concerned that the flashed transient could have disrupted ongoing processing in some way and that this disruption was affecting TTC estimates. Consistent with this notion, when the flashed marker was replaced by a static marker, we found less general disruption of the representational-momentum distributions (proportion of same responses) and a much clearer relationship between representational momentum and the change in perceived TTC. 
The correlations between representational momentum and TTC for individual observers suggest two things. First, they provide further evidence of a positive, albeit fairly weak, relationship between these two effects. Second, they reiterate the point that other factors are also important modulators of these effects. For instance, representational momentum is a fairly poor predictor of initial TTC. This suggests that in addition to localization of initial point of disappearance, other factors, such as extrapolation errors or timing errors (DeLucia and Liddell 1998) help determine TTC estimates. Similarly, the lengthening of perceived TTC produced by disambiguating the point of disappearance, while possibly related to the extent of observed representationalmomentum shift, is clearly influenced by other factors that seem to make the current participants more or less susceptible to the display manipulations. Given the current set of data, we are unable to determine whether such other factors are task specific, having to do with the particular way individual observers approach the problem of TTC estimation, or are more general in nature.

Peterken et al (1991 - experiment 3; condition 4) previously examined the effect of a stationary reference frame on TTC judgments using a PM paradigm and found no significant differences in TTC estimates. We now consider some possible reasons for these conflicting results. One important difference between the two studies was the instructions given to observers for the TTC task. Peterken et al instructed their observers to track the moving target with eye movements while observers in the present experiment were instructed to fixate the center of the screen. While in a separate experiment Peterken et al did show that there was no significant difference in TTC estimation when subjects were instructed to track the target versus when they were instructed to fixate, they did not compare these two instructions when the stationary reference frame was used. It is possible that a reference frame has more of an effect on TTC estimates when there is no eye tracking because presumably it would be more difficult to extrapolate the motion of the target after it disappeared and instead observers must use either the $\tau$ strategy or the distance/velocity strategy. Indeed, the observers in the Peterken et al experiment reported that the task seemed more difficult when eye tracking was prevented. It is also possible that pairing the TTC and representational-momentum tasks in the present experiment influenced the observers' strategy. Another important difference between the present study and the work by Peterken et al could be the structure of the reference frame. In the present experiment, we used elongated vertical teeth that were very close to the moving target while the markers in the Peterken et al study were small dashes that were a greater distance from the moving target (the exact dimensions of their frame were not provided). Given that there was such a larger difference between the teeth and tunnel conditions in the present experiment it would seem that TTC estimates are very sensitive to the particular structure of the reference frame.

\section{General discussion}

In two experiments we have demonstrated that memory for the final visible position of a moving object plays an important role in determining TTC estimates. Specifically, under conditions in which the remembered final location of a moving object is shifted forward in the direction of motion, a phenomenon known as representational momentum (Freyd and Finke 1984), TTC was shown to be consistently underestimated. Such underestimation is a very well established finding in the TTC literature (eg Cavallo and Laurent 1988; McLeod and Ross 1983; Schiff and Detwiler 1979; Stewart et al 1993). In conditions where the point of disappearance was disambiguated, either by flashing a brief marking grid (experiment 1) or by providing a spatially salient context (experiment 2), underestimates of TTC and the magnitude of representational momentum were substantially reduced. 
The first implication of these findings is that the initial point of disappearance is an important variable in determining TTC. In section 1 we discussed different perceptual strategies that have been put forth to explain how observers might perform the TTC judgment in the occlusion paradigm. Given that these TTC strategies are all affected slightly differently by disambiguating the disappearance point, manipulations like those described here may be an effective tool for investigating the different quantitative models of TTC estimation (eg Gray and Regan 1999b; Rushton and Wann 1999) in future experiments.

Finally, we want to address two issues relating specifically to representational momentum. There can be little doubt that under unmarked conditions observers in experiments 1 and 2 have biased estimates of the point of disappearance (see figures 2 and 5). Our interpretation of this bias has been framed within the context of representational momentum and, more specifically, dynamic mental representations (Freyd 1987). However, in the current displays, other factors, such as tracking errors or eye movement artifacts may also contribute to the size of the bias (Kerzel et al 1999). While such oculomotor effects cannot provide a complete explanation for the general range of representational-momentum findings (see Hubbard 1995a for a discussion), here they certainly predict a similar forward bias. However, even if the explanatory power of representational momentum was questioned in the current context, our main claim - that localization of the vanishing point influences TTC estimates-would be unaffected.

It is also important to reiterate that we are not proposing representational momentum as the sole cause of TTC errors. This is clearly not the case. In the current data set, while all thirty-two observers had very similar representational-momentum shifts, twenty-four underestimated TTC while eight strongly and consistently overestimated TTC. In addition to errors caused by mislocalization of the point of disappearance, errors in TTC estimation could arise from clocking errors, errors in the extrapolation process, or eye movement tracking errors (Haywood 1977). It may be differences in these mechanisms that account for why some observers overestimate and others underestimate.

Our suggestion is simply that, whatever technique observers use to compute TTC, they almost certainly rely on some internal representation of the object and/or its behavior. If there are systematic biases in this representation, then we may be able to detect links between the two phenomena. It is interesting to note that while there are differences in the initial sign of the TTC error, all observers show a consistent change in behavior, that is an increase in estimates of TTC in the marked condition. A common internal representation system used by different mechanisms could account for this pattern of results.

Acknowledgements. The authors contributed equally to this research, and authorship order was determined arbitrarily. Thanks are extended to Ellen Hildreth, Ron Rensink, and three anonymous reviewers for commenting on an earlier draft of this manuscript.

\section{References}

Bootsma R J, 1989 "Accuracy of perceptual processes subserving different perception action systems" Quarterly Journal of Experimental Psychology A $41489-500$

Bootsma R J, Oudejans R R, 1993 "Visual information about time-to-collision between two objects" Journal of Experimental Psychology: Human Perception and Performance 191041 - 1052

Brehaut J C, 1989 The Role of Attention in Representational Momentum unpublished manuscript, Mount Allison University, Sackville, New Brunswick, Canada

Brehaut J C, Tipper S P, 1996 "Representational momentum and memory for luminance" Journal of Experimental Psychology: Human Perception and Performance 22 480-501

Brown J F, 1931 "The visual perception of velocity" Psychologische Forschung 14 199-232

Cavallo V, Laurent M, 1988 "Visual information and skill level in time-to-collision estimation" Perception $17623-632$ 
Cooper L A, 1989 "Mental models of the structure of visual objects", in Object Perception: Structure and Process Eds B E Shepp, S Ballesteros (Hillsdale, NJ: Lawrence Erlbaum Associates) pp $91-119$

DeLucia P R, Liddell G W, 1998 "Cognitive motion extrapolation and cognitive clocking in prediction motion tasks" Journal of Experimental Psychology: Human Perception and Performance $24901-914$

Faust M E, 1990 Representational Momentum: A Dual Process Perspective unpublished doctoral dissertation, University of Oregon, Eugene, OR, USA

Finke R A, Freyd J J, Shyi G C-W, 1986 "Implied velocity and acceleration induce transformations of visual memory" Journal of Experimental Psychology: General 115 175-188

Finke R A, Shyi G C-W, 1988 "Mental extrapolation and representational momentum for complex implied motions" Journal of Experimental Psychology: Learning, Memory, and Cognition $14112-120$

Freyd J J, 1987 "Dynamic mental representations" Psychological Review 94 427-438

Freyd J J, 1992 "Dynamic representations guiding adaptive behavior", in Time, Action and Cognition: Towards Bridging the Gap Eds F Macar, V Pouthas, W J Friedman (Dordrecht: Kluwer Academic Publishers) pp 309-323

Freyd J J, Finke R A, 1984 "Representational momentum" Journal of Experimental Psychology: Learning, Memory, and Cognition $10126-132$

Freyd J J, Finke R A, 1985 "A velocity effect for representational momentum" Bulletin of the Psychonomic Society $23443-446$

Freyd J J, Johnson J Q, 1987 "Probing the time course of representational momentum" Journal of Experimental Psychology: Learning, Memory, and Cognition 13 259-268

Freyd J J, Jones K T, 1994 "Representational momentum for a spiral path" Journal of Experimental Psychology: Learning, Memory, and Cognition $20968-976$

Gogel W C, McNulty P, 1983 "Perceived velocity as a function of reference mark density" Scandinavian Journal of Psychology 24 257-265

Gray R, Regan D, 1998 "Accuracy of estimating time to collision using binocular and monocular information" Vision Research $38499-512$

Gray R, Regan D, 1999a "Do monocular time-to-collision estimates necessarily involve perceived distance?" Perception $281257-1264$

Gray R, Regan D, 1999b "Motion in depth: Adequate and inadequate simulation" Perception \& Psychophysics $61236-245$

Hayes A, 1997 Representational Momentum under Conditions of Divided Attention unpublished doctoral dissertation, University of Oregon, Eugene, OR, USA

Hayes A, Freyd J J, 1995 "Attention and representational momentum", technical report no 95-12, Institute of Cognitive and Decision Sciences, University of Oregon, OR, USA

Hayes A, Sacher G, Thornton I M, Sereno M E, Freyd J J, 1996 "Representational momentum in depth using stereopsis" Investigative Ophthalmology \& Visual Science 37(4) S3

Hayes A E, Thornton I M, 1999 "Are we lost in space during ego-motion?" Investigative Ophthalmology \& Visual Science 40(4) S4212

Haywood K M, 1977 "Eye movements during coincidence-anticipation performance" Journal of Motor Behavior $9313-318$

Hubbard T L, 1990 "Cognitive representations of linear motion: possible direction and gravity effects in judged displacements" Memory \& Cognition 18 299-309

Hubbard T L, 1993 "The effect of context on visual representational momentum" Memory \& Cognition $21103-114$

Hubbard T L, 1995a "Environmental invariants in the representation of motion: Implied dynamics and representational momentum, gravity, friction and centripetal force" Psychonomic Bulletin \& Review $2322-338$

Hubbard T L, 1995b "Displacement in depth: Representational momentum and boundary extension" Psychological Research/Psychologische Forschung $5933-47$

Hubbard T L, 1996 "Representational momentum, centripetal force, and curvilinear impetus" Journal of Experimental Psychology: Learning, Memory, and Cognition 22 1049-1060

Hubbard T L, Bharucha J J, 1988 "Judged displacement in apparent vertical and horizontal motion" Perception \& Psychophysics 44211 - 221

Hubbard T L, Ruppel S E, 1999 "Representational momentum and landmark attraction effects" Canadian Journal of Experimental Psychology $53242-256$

Jagacinski R, Johnson W, Miller R, 1983 "Quantifying the cognitive trajectories of extrapolated movements" Journal of Experimental Psychology: Human Perception and Performance $943-57$ 
Kelly M H, Freyd J J, 1987 "Explorations of representational momentum" Cognitive Psychology $19369-401$

Keppel G, 1991 Design and Analysis: A Researcher's Handbook (Englewood Cliffs, NJ: PrenticeHall)

Kerzel D, Jordan J S, Müsseler J, 1999 "Testing a perceptual-oculomotor account of effects deemed due to representational momentum", paper presented at the 40th Annual Meeting of the Psychonomic Society, Los Angeles, CA, USA

Lee D N, 1976 "A theory of visual control of braking based on information about time to collision" Perception 5 437-459

Lyon D R, Waag W L, 1995 "Time course of visual extrapolation accuracy" Acta Psychologica $89239-260$

McLeod R W, Ross H E, 1983 "Optic flow and cognitive factors in time-to-collision estimates" Perception $12417-423$

Munger M P, Solberg J L, Horrocks K K, Preston A S, 1999 "Representational momentum for rotations in depth: Effects of shading and axis" Journal of Experimental Psychology: Learning, Memory, and Cognition $25157-171$

Peterken C, Brown B, Bowman K, 1991 "Predicting the future position of a moving target" Perception $205-16$

Prinzmetal W, Wilson A, 1997 "The effect of attention on phenomenal length" Perception 26 $193-205$

Reed C L, Vinson N G, 1996 "Conceptual effects on representational momentum" Journal of Experimental Psychology: Human Perception and Performance 22 839-850

Rosenbaum D A, 1975 "Perception and extrapolation of velocity and acceleration and velocity" Journal of Experimental Psychology: Human Perception and Performance 1395-403

Rushton S K, Wann J P, 1999 "Weighted combination of size and disparity: a computational model for timing a ball catch" Nature Neuroscience 2 186-190

Savelsbergh G J P, Whiting H T A, Bootsma R J, 1991 "Grasping tau” Journal of Experimental Psychology: Human Perception and Performance 17 315-322

Schiff W, Detwiler M L, 1979 "Information used in judging impending collision" Perception 8 $647-658$

Stewart D, Cudworth C J, Lishman J R, 1993 "Misperception of time-to-collision by drivers in pedestrian accidents" Perception $221227-1244$

Thornton I M, DiGirolamo G J, Hayes A, Freyd J J, 1996 "Representational momentum under conditions of visual distraction" Abstracts of the Psychonomic Society 140

Tresilian J R, 1995 "Perceptual and cognitive processes in time-to-contact estimation: analysis of prediction-motion and relative judgment tasks" Perception \& Psychophysics $57231-245$

Verfaillie K, d'Ydewalle G, 1991 "Representational momentum and event course anticipation in the perception of implied periodical motions" Journal of Experimental Psychology: Learning, Memory, and Cognition $17302-313$

Zakay D, Block R A, 1997 "Temporal cognition" Current Directions in Psychological Science 6 $12-16$ 